\title{
Miniesternotomía para cirugía valvular aórtica: Experiencia inicial en un centro cardiovascular en Chile
}

\author{
Juan Carlos Bahamondes S, G ustavo Meriño S, \\ Juan Salman A, Abelardo Silva V, Jean Pierre D roguett G. \\ Ministernotomy for aortic valve \\ surgery: Report of 20 patients
}

Background: Aortic valve surgery can be performed through a reduced mid sternotomy with excellent long term results. Aim: To report the initial results obtained with this technique. Patients and methods: Descriptive study of 20 patients aged $48 \pm 11$ years, subjected to valve replacement surgery for aortic valve disease between 2004 and 2007. Arterial and venous cannulation were performed with the usual method and extracorporeal circulation was performed with a mean perfusion of $4.5 \mathrm{~L} / \mathrm{min}$. Hypothermia and cardioplegia were performed infusing the hematic cardioplegic solution at $4^{\circ} \mathrm{C}$ in the aortic root or coronary ostia. Results: Sixteen patients were in functional class (FC) III. Fourteen patients had aortic insufficiency and six had predominant stenosis. There was no operative mortality. One patient had a left hemothorax and was reoperated. All patients were discharged between 4 and 6 days after surgery. Mean follow up was $21 \pm 4$ months. All patients are in FC I and free from cardiac events. Echocardiographic assessment was done in 16 patients, showing a good motility of valve disks. Actuarial survival probability was $100 \%$ and probability of freedom from cardiac events was $100 \%$ at 42 months of follow up. Conclusions: Ministernotomy is an excellent approach for aortic valve surgery providing good visualization of the ascending aorta, simplifying the surgical technique (Rev Méd Chile 2008; 136: 1141-6).

(Key words: Aortic valve; Cardiac surgical procedures; Heart valve diseases; Surgical procedures, minimally invasive)

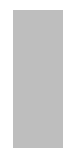

Recibido el 4 de octubre, 2007. Aceptado el 29 de mayo, 2008.

Este estudio no tuvo apoyo financiero.

Unidad de Cirugía Cardiovascular, Centro Cardiovascular, Hospital Regional de Temuco. Departamento de Cirugía, Facultad de Medicina, Universidad de La Frontera. Temuco, Chile.

T a esternotomía media fue por primera vez descrita en 1897 por Milton ${ }^{1}$, utilizada para resección de linfonodos tuberculosos. En cirugía

$\overline{\text { Correspondencia a: Dr. Juan Carlos Bahamondes S. Depar- }}$ tamento de Cirugía, Universidad de La Frontera. Av.

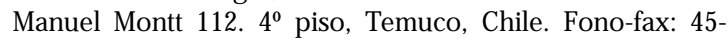
325760. E mail: jcbahamo@ufro.cl cardiaca este acceso fue introducido por Julian et $\mathrm{al}^{2}$ en 1957, sustituyendo a la toracotomía anterior bilateral logrando un gran beneficio a un sinnúmero de pacientes de la época, por reducir el tiempo de cirugía, favorecer una excelente exposición del corazón y disminuir el trauma respiratorio.

Desde esos años, la esternotomía media ha sido el acceso de elección en cirugía cardiaca, ofreciendo 
ésta un amplio campo quirúrgico al exponer cámaras cardiacas y grandes vasos. Pero con la introducción de técnicas mínimamente invasivas en cirugía coronaria en la década anterior, las cuales se extendieron a la cirugía de la válvula aórtica, ha existido un interés mundial en utilizar accesos reducidos con la premisa de disminuir el trauma quirúrgico, el tiempo de hospitalización y obtener mejores resultados estéticos para los pacientes. En 1996, Cosgrove y Sabik ${ }^{3}$ de Cleveland Clinic introducen la toracotomía longitudinal paraestemal en cirugía valvular aórtica, comunicando las ventajas estéticas, la estabilidad torácica y la disminución del sangrado post operatorio e infecciones de la herida esternal. La cirugía valvular mediante miniestemotomía en J introducida por Svensson ${ }^{4,5}$ es una nueva alternativa para la cirugía de la válvula aórtica y que está aún en discusión en algunos grupos quirúrgicos, ya que ha demostrado conseguir los mismos resultados que la técnica convencional, los cuales son el objetivo de esta comunicación.

\section{PACIENTES Y MÉTODO}

Pacientes. Entre junio de 2004 y abril de 2007, se realizó reemplazo valvular aórtico en 78 pacientes consecutivos en forma electiva. En 20 pacientes se accedió al corazón mediante una miniesternotomía media, siendo éste el grupo de nuestro estudio. Los 58 pacientes restantes fueron intervenidos mediante esternotomía media convencional y constituyen el grupo control. La decisión de la utilización de miniesternotomía fue efectuada por el cirujano el día de la intervención, quien seleccionó a pacientes mesomórficos sin distingo por edad o por predominio de estenosis o insuficiencia aórtica.

La edad promedio fue 48,1 años ( $\mathrm{DE} \pm 17,65$ ) con un rango entre 32 a 67 años. La distribución por sexo fue similar. En el período preoperatorio 16 pacientes estaban en CF III, según la clasificación NYHA. En cuanto al mecanismo de lesión valvular, 14 pacientes se presentaron con predominio de insuficiencia aórtica y los 6 restantes tenían predominio de estenosis (Tabla 1).

Método. Estudio descriptivo de pacientes en quienes se efectuó reemplazo valvular aórtico mediante miniesternotomía, identificados mediante la base de datos de cirugía cardiovascular de nuestro centro. Se revisaron las fichas clínicas y protocolos operatorios. En el estudio preoperatorio, se realizó ecocardiografía a todos los pacientes y coronariografía en mayores de 50 años. Para el seguimiento se utilizaron datos de las fichas clínicas, controles

\section{Tabla 1. Variables biodemográficas de pacientes intervenidos por miniesternotomía} y esternotomía convencional

\begin{tabular}{|lccc|}
\hline Variable & $\mathrm{n}=20$ & $\mathrm{n}=58$ & \\
\hline Edad (años) & & & $\mathrm{p}=0,87$ \\
$\quad$ Promedio \pm DE & $48,1 \pm 15,51$ & $52,2 \pm 12,34$ & \\
$\quad$ Rango & $32-67$ & $36-77$ & $\mathrm{p}=0,56$ \\
Sexo & & & $\mathrm{p}=0,62$ \\
$\quad$ Masculino & 10 & 25 & \\
Femenino & 10 & 33 & $\mathrm{p}=0,85$ \\
NYHA CF & 0 & 0 & $\mathrm{p}=0,74$ \\
I & 4 & 4 & \\
II & 16 & 54 & $\mathrm{p}=0,58$ \\
III & 0 & 0 & $\mathrm{p}=0,67$ \\
IV & 6 & 20 & $\mathrm{p}=0,87$ \\
Patología & 14 & 38 & 4,9 \\
$\quad$ Estenosis & 4,6 & & \\
Insuficiencia & & & \\
EuroSCORE & & & \\
\hline
\end{tabular}


en policlínico, datos obtenidos en el registro civil, o mediante contacto telefónico con el paciente.

Técnica quirúrgica. Previa inducción de la anestesia general, el paciente es posicionado en decúbito supino instalando parches autoadhesivos para desfibrilación externa en la región dorsal. El campo quirúrgico es preparado con técnica estéril en forma habitual. Se accede al tórax mediante una incisión cutánea en la línea media no mayor a 8-10 cm, ubicando el ángulo de Louis y luego midiendo $2 \mathrm{~cm}$ sobre este punto de reparo hacia la incisura esternal. El resto de la incisión se prolonga los 6 a $8 \mathrm{~cm}$ restantes hacia el cuerpo esternal (Figura 1).

El hueso esternal es escindido mediante sierra neumática desde el yugulum hasta la altura del

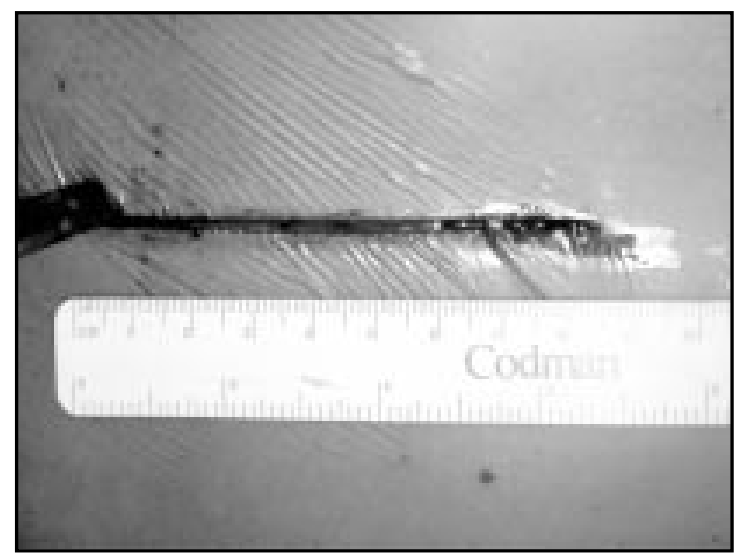

Figura 1. Incisión para miniesternotomía de $8 \mathrm{~cm}$.

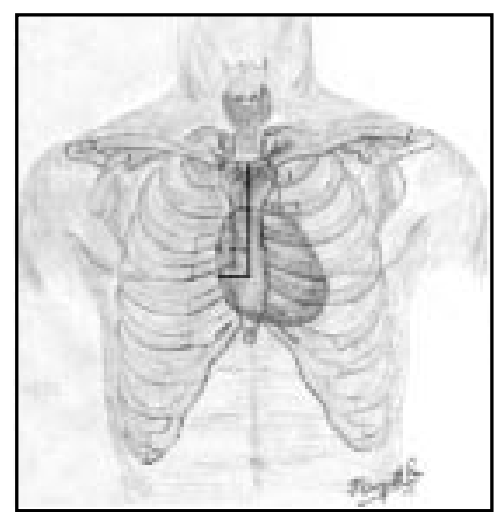

Figura 2. Diagrama miniesternotomía en 3o o $4^{\circ}$ espacio intercostal. tercer o cuarto espacio intercostal. A ese nivel, la esternotomía se prolonga transversalmente al tercer o cuarto espacio intercostal derecho, teniendo precaución de no dañar la arteria mamaria interna derecha (Figura 2). Posteriormente, el tejido graso y remanente tímico del mediastino anterior es seccionado y ligado para evitar sangrados posteriores. El pericardio es abierto en la línea media en forma de $\mathrm{T}$ invertida, asegurándolo mediante suturas ajustadas al borde de la incisión, con lo cual se logra una excelente exposición de la aorta ascendente en todo su trayecto y aurícula derecha. Luego de la heparinización sistémica, la circulación extracorpórea (CEC) es establecida mediante canulación en aorta ascendente o cayado proximal y cánula venosa única en la orejuela de la aurícula derecha, utilizando hipotermia sistémica moderada a $30^{\circ} \mathrm{C}$.

El pinzamiento aórtico se realiza mediante un clamp de Fogarty. Las cavidades izquierdas son drenadas mediante un vent aurículo-ventricular introducido a través de la vena pulmonar superior derecha o mediante un aspirador directo en el ventrículo izquierdo a través del anillo aórtico durante el periodo de extracción y aseo valvular. La protección miocárdica se efectúa mediante solución de cardioplegia hemática a $4^{\circ} \mathrm{C}$, infundida en forma intermitente cada 20 min en la raíz aórtica en los casos de predominio de estenosis aórtica y en los ostia coronarios en forma directa, luego de abrir la aorta en los pacientes en que predominó la insuficiencia aórtica. En todos los pacientes se realizó aortotomía oblicua permitiendo una excelente exposición de la válvula aórtica y ostia coronarios mediante suturas de tracción hacia craneal combinadas con tracción de las comisuras. La válvula es extraída completamente, decalcificando luego el anillo aórtico. La prótesis es suturada en el anillo mediante suturas de Tycron 2/0, visualizando ambos ostia coronarios al completar el procedimiento (Figura 3). La aortotomía es cerrada con sutura continua no reabsorbible 4/0. Se remueve el aire de las cavidades y se procede al despinzamiento aórtico y retiro de CEC. Luego de revisada la hemostasia, se instalan cables de marcapaso epicárdicos y un drenaje mediastínico en la línea media.

Estadística. Se utilizó estadística descriptiva con medidas de tendencia central y dispersión; test 


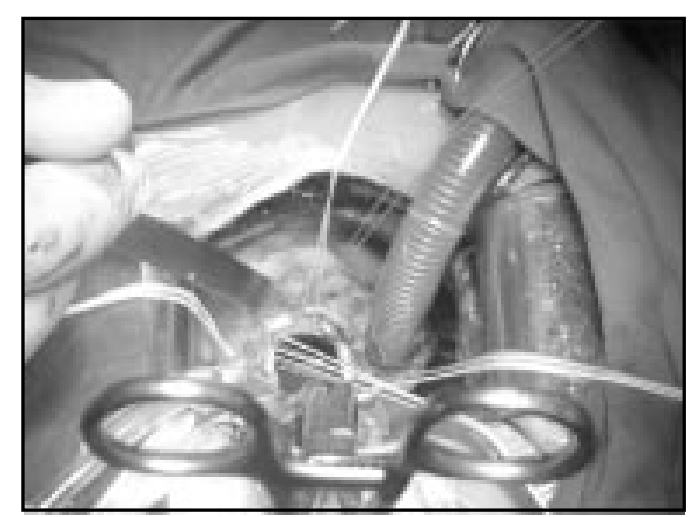

Figura 3. Reemplazo valvular aórtico mediante miniesternotomía.

exacto de Fisher y $\mathrm{chi}^{2}$ de Pearson para variables dicotómicas y t-test para variables continuas. Se consideró estadísticamente significativo un valor $\mathrm{p}$ $<0,05$. Se utilizó el test de ANOVA para comparación de varianzas, y el método de Kaplan-Meier para el cálculo de supervivencia actuarial, con el programa Stata $9.2^{\circledR}$.

\section{Resultados}

Los resultados se expresan en medidas de tendencia central. En todos los casos se consiguió una buena exposición del plano valvular, lo que permitió el reemplazo protésico sin contratiempos. El acceso esternal varió entre 8 y $10 \mathrm{~cm}$, con un promedio de $9 \mathrm{~cm}$. Todos fueron realizados en J con prolongación al cuarto o quinto espacio intercostal derecho. El tiempo de CEC y clamp aórtico fue en promedio de $70 \mathrm{~min}(\mathrm{DE} \pm 5,75)$ y 58 min $(\mathrm{DE} \pm 3,56)$, respectivamente, alcanzando un flujo de CEC de 4,5 1/min en promedio, no alcanzando diferencias estadísticamente significativas con el grupo control. En todos los pacientes se implantó una prótesis mecánica Saint Jude. El tiempo promedio de ventilación mecánica fue de 6,3 h (5-12 h), el cual fue menor que para el grupo con esternotomia convencional. La estadía postoperatoria en Unidad de Cuidados Intensivos (UCI) y la cantidad de días de hospitalización fue menor en el grupo intervenido mediante miniesternotomía. El sangrado postoperatorio y el número de transfusiones fueron menores para la miniesternotomía, alcanzando diferencias estadísticamente significativas como es posible apreciar en la Tabla 2.

La exposición quirúrgica fue satisfactoria en todos los casos, no necesitándose conversión a esternotomía media. No hubo complicaciones directamente relacionadas con el tipo de acceso (seromas, hematomas, inestabilidad esternal, in-

Tabla 2. Comparación de pacientes intervenidos mediante miniesternotomía y esternotomía convencional

\begin{tabular}{|lccc|}
\hline Variable & $\mathrm{n}=20$ & $\mathrm{n}=58$ & $\mathrm{p}$ \\
\hline Tiempo CEC (min) promedio & $70 \pm 5,75$ & $78 \pm 4,31$ & $\mathrm{p}=0,73$ \\
Tiempo clamp (min) promedio & $58 \pm 3,56$ & $60 \pm 5,64$ & $\mathrm{p}=0,67$ \\
Ventilación mecánica (h) & $6,3(5-12)$ & $8,1(6-15)$ & $\mathrm{p}=0,48$ \\
№ días UCI promedio & $2,2(2-4)$ & $3,8(3-5)$ & $\mathrm{p}=0,53$ \\
№ días hospitalización promedio & $6,2(4-7)$ & $7,8(7-10)$ & $\mathrm{p}=0,53$ \\
Sangrado (ml) promedio & $450 \pm 56,6$ & $633 \pm 75,4$ & $\mathrm{p}=0,08$ \\
Transfusión de GR (no de unidades) promedio & $2 \pm 1,25$ & $4 \pm 3,34$ & $\mathrm{p}=0,05$ \\
\hline
\end{tabular}


fecciones, cicatriz hipertrófica). Un paciente presentó fibrilación auricular transitoria que requirió manejo farmacológico y otro presentó un hemotórax que requirió reexploración y drenaje a través de la miniesternotomía. No hubo mortalidad perioperatoria en nuestra serie.

Seguimiento. El seguimiento se cerró en diciembre de 2007 y fue completo en todos los casos con un promedio de 20,9 meses ( $\mathrm{DE} \pm 3,92$ ), esto debido al poco tiempo de iniciada la técnica en nuestro centro. Todos los pacientes se encuentran en CF I y libres de eventos cardiovasculares en el período observado. Se realizó ecocardiografía de control en 16 pacientes, evidenciándose una buena movilidad de los discos protésicos, no hubo deterioro de la función ventricular preoperatoria y no se demostró derrame pericárdico. La probabilidad de supervivencia actuarial es de $100 \%$, y la probabilidad de supervivencia actuarial libre de eventos cardiovasculares fue de $100 \%$ a 42 meses. Durante este periodo no se observó ninguna secuela estética derivada de la cicatriz de la herida operatoria y si bien no se utilizó ninguna escala de satisfacción del procedimiento, éste fue catalogado como de excelente resultado estético por la mayoría de los pacientes.

\section{DisCUSIÓN}

La esternotomía media clásica es indiscutiblemente el acceso que permite una visión completa de las estructuras cardiacas durante la cirugía con circulación extracorpórea. Pero a la vez, produce un trauma quirúngico mayor al tener una incisión amplia en la piel desde la horquilla esternal hasta el apéndice xifoides y como también el estar asociada a distensión y rotura de estructuras ligamentosas del esternón en relación a la inserciones costales, todo lo cual se traduce en un mayor dolor postoperatorio, una recuperación más lenta y secuelas estéticas permanentes ${ }^{3-5,7}$. Desde el inicio de la miniesternotomía como técnica, se aprecia un gran interés en los distintos grupos quirúrgicos de cirugía cardiaca a nivel mundial por realizar procedimientos que tiendan a reducir la agresión quirúrgica, ya que se han minimizado las complicaciones derivadas de la esternotomía media ${ }^{7}$. Después de una gran expe- riencia preliminar con la esternotomía convencional, escogimos a la miniesternotomía en J como acceso alternativo en cirugía de la válvula aórtica, debido a que permite una visualización directa de la aorta y estructuras cardiacas derechas, haciendo posible la utilización de instrumental y canulación estándar, con un riesgo quirúrgico similar según lo demuestra el EuroSCORE promedio del grupo en comparación con el grupo control.

En nuestro medio destaca la experiencia comunicada por el grupo de la Universidad Católica de Chile $^{8}$ que ha demostrado excelentes resultados, con incisiones de longitud similar a la expuesta en la literatura y en nuestro centro, sin necesidad de convertir a estemotomía media ${ }^{3-6}$. A nivel mundial, estudios prospectivos y de distribución aleatoria demuestran resultados estadísticamente contradictonos en cuanto a sangrado, estadía hospitalaria y resultados cosméticos ${ }^{9,10}$, pudiendo estar limitados probablemente por el número de pacientes incluidos en dichos estudios. En nuestros pacientes se objetivó un menor sangrado y menor uso de transfusiones en el peníodo postoperatorio con diferencias estadísticamente significativas en relacion al grupo con esternotomía convencional, lo que en parte demuestra un menor trauma quirúrgico en estos pacientes. Cohn et $\mathrm{al}^{11}$ encontraron $27 \%$ de fibrilación auricular postoperatoria en pacientes sometidos a reemplazo valvular aórtico a través de miniestemotomía y Weinschelbaum et $\mathrm{al}^{12} 12,6 \%$ a través del acceso paraesternal. Nuestra serie reporta un caso, correspondiendo éste a 6,25\%, probablemente debido al número bajo de pacientes incluidos en este estudio. La estadía hospitalaria fue similar a otras publicaciones $3,4,10,11$, fluctuando entre 4 y 6 días, no existiendo diferencias estadísticamente significativas con respecto a la esternotomía media ${ }^{10,13-15}$.

En base a lo anterior, se puede concluir que la miniesternotomía en J utilizada para la cirugía de la válvula aórtica es una excelente altemativa de acceso, consiguiendo un campo operatorio satisfactorio y al mismo tiempo mantiene la eficiencia y eficacia de la cirugía clásica, al permitir una completa visualización de la raíz y aorta ascendente con una reducción en el traumatismo estemal y sin producir un aumento en el tiempo quirúrgico total. Además, esta técnica no sólo provee resultados satisfactorios para los pacientes, los cuales son comparables con la técnica convencional, sino que además posibilita una recuperación precoz y con secuelas mínimas. 


\section{REFERENCIAS}

1. Mitron H. Mediastinal surgery. Lancet 1897; 1: 872-5.

2. Julan OC, López-Belo M, Dye WS. The median sternal incision in intracardiac surgery with extracorporeal circulation: a general evaluation of its use in heart surgery. Surgery 1957; 42: 753-61.

3. Cosgrove III DM, SABIK JF. Minimally invasive approach for aortic valve operations. Ann Thorac Surg 1996; 62: 596-7.

4. Cosgrove III DM, Navia JL, SABiK JF. Minimally invasive valve operations. Ann Thorac Surg 1998; 65: 1535-9.

5. Svensson LG, D'Agostino RS. "J" incision minimalaccess valve operations. Ann Thorac Surg 1998; 66: 1110-2.

6. Svensson LG, D'Agostino RS. Minimal-access aortic and valvular operations, including the "J" incision. Ann Thorac Surg 1998; 66: 431-5.

7. Mestres CA, Belda J, Greco E, Cartana R, Gimferrer JM. Combined lung resection and aortic valve replacement via ministernotomy. Asian Cardiovasc Thorac Ann 2001; 9: 155-6.

8. Zalaquett R, Baeza C, Irarrázaval M, Morán S, Becker P, MatuRANa G et aL. Esternotomía mínima en cirugía valvular. Rev Chil Cardiol 1999; 18: 63-8.
9. Bonacchi M, Prifti E, Giunti G, Frati G, Sani G. Does ministernotomy improve postoperative outcome in aortic valve operation? A prospective randomized study. Ann Thorac Surg 2002; 73: 460-5.

10. Aris a, Camara ML, Montiel J, Delgado Lj, Galan J, LTVAN H. Ministernotomy versus median sternotomy for aortic valve replacement: a prospective, randomized study. Ann Thorac Surg 1999; 67: 1583-7; discussion 1587-8.

11. Cohn LH, Adams DH, Couper GS. Minimally invasive cardiac valve surgery improves patient satisfaction while reducing costs of valve replacement and repair. Ann Surg 1997; 226: 421-6.

12. Weinschelbaum E, Stutzbach P, Machain A, Favaloro R, Caramutti V, Bertolotti A, Fraguas H. Valve operations through a minimally invasive approach. Ann Thorac Surg 1998; 66: 1106-9.

13. Aris A, Padró JM, Cámara ML. Sustitución valvular aórtica mínimamente invasiva. Rev Esp Cardiol 1997; 50: 750-3.

14. Cooley D. Minimally invasive valve surgery versus the conventional approach. Ann Thorac Surg 1998; 66: 1101-5.

15. Szwerc MF, Benckart DH, Wiechmann RJ, Savage EB, Szydlowski GW, Magovern Jr GJ, Magovern JA. Partial versus full sternotomy for aortic valve replacement. Ann Thorac Surg 1999; 68: 2209-13. 